\title{
Thanks to all those who reviewed for Biological Procedures Online in 2014
}

Shulin Li

\section{Contributing reviewers}

A peer-reviewed journal would not survive without the generous time and insightful comments of the reviewers, whose efforts often go unrecognized. Although final decisions are always editorial, they are greatly facilitated by the deeper technical knowledge, scientific insights, understanding of social consequences, and passion that reviewers bring to our deliberations. For these reasons, the Editor-in-Chief and staff of the journal warmly thank the reviewers whose comments helped to shape Biological Procedures Online, for their invaluable assistance with review of manuscripts for the journal in Volume 16 (2014).

\author{
Aruna Ambagala \\ Canada
}

Hosam Arafate

Saudi Arabia

Deb Court

Canada

Marina Cretich

Italy

Wim Dhaeze

USA

Marxa Figueiredo

USA

Ching-Hung Hsu

USA

Ching-Hung Hsu

China

\author{
Yingqi Hua \\ USA
}

Michael Hust

Germany

David le Couteur

Australia

Bolin Liu

USA

Lucia Manso-Silvan

France

Nitish Mishra

USA

Mauro Provinciali

Italy

Sebastien Rodrigue

Canada
Lucia Sacchetti

Italy

Akinori Sato

Japan

Masatoshi Tagawa

Japan

Lynn Thomason

USA

Shi Xu

USA

Shaomian Yao

USA

Correspondence: Sli4@mdanderson.org

Department of Pediatrics-Research, Unit 0853, The University of Texas MD

Anderson Cancer Center, 1515 Holcombe Blvd., Houston, TX 77030, USA 